\title{
On the design of waveguide-to-microstrip and waveguide-to-coplanar line transitions
}

\author{
Jan Machac*, Wolfgang Menzel** \\ * Faculty of Electrical Engineering, Czech Technical University, \\ Technicka 2; 16627 Prague 6. Czech Republic. Tel.: (++422) \\ 332 2299; Fax: $(++422) 311$ 1786; e-mail: MACHAC@ \\ CSPUNI 12 . bitnet \\ * * University of Ulm, Albert Einstein Allee 41,7900 Ulm, \\ Germany; Tel.: (++497311502 6350; Fax: $1++49731$ 1) 502 \\ 6359; e-mail: MENZEL@ mwt.e-technik.uni-ulm.de
}

\section{Abstract}

New versions of the waveguide-to-microstrip and waveguide-tocoplanar line transitions are described and analyzed. The transitions differ from the standard ones by a probe in the shape of a rectangular patch. The structure is very simple, easily adjustable, it has a broad bandwidth, and there is no need to use an impedance transformer. These transitions have been analyzed by the method of minimal autonomous blocks and the integral equation method. The results obtained were compared with experimental data. An optimized structure has been designed by a simple optimization process using the integral equation method. Its transition band is flat with return losses better than $-15 \mathrm{~dB}$ nearly in the whole X-band.

\section{Introduction}

There are three well-known types of waveguide-to-microstrip transitions: a ridged-waveguide taper, a finline taper and an Eplane probe [1]. Only a few of information can be found in the literature concerning the E-plane probe transition $[2,3]$. Without an impedance transformer, its transition bandwidth is rather narrow. The new structure with a rectangular patch instead of a strip probe has significantly broader bandwidth. This new transition type has been designed both in a microstrip version (Fig.la) and, changing slightly the geometry, in a coplanar line version (Fig. Ib). There is no need to use an impedance transformer in this transition type. The best performance is adjusted by a proper backshort position.

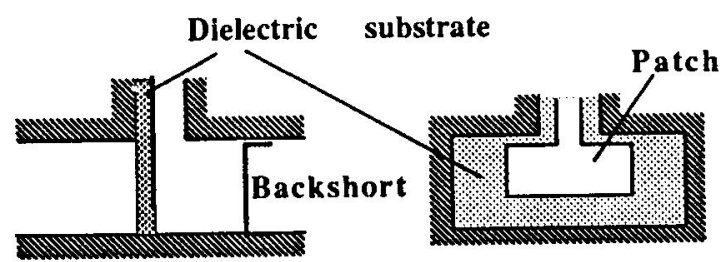

$\mathbf{a}$

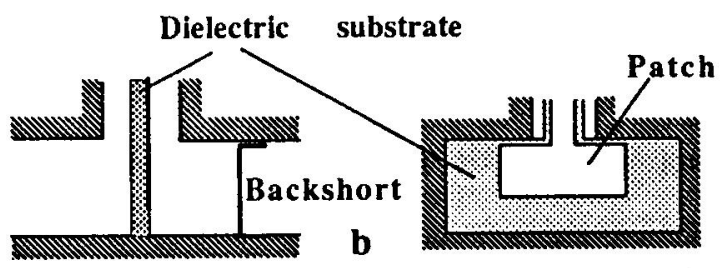

Fig.1: Transition structures: a) microstrip version b) coplanar version

The E-plane waveguide-to-microstrip transition with dielectric substrate parallel to the waveguide axis and with infinitesimally narrow probe strip was solved in [3] using the spectral-domain technique. This is not applicable for the structure under consideration where the probe has the shape of a rectangular patch perpendicular to the waveguide axis.
The structures were analyzed using the integral equation method [4] in comparison with the method of minimal autonomous blocks (MAB) [5]. Several structures with different geometry and patch dimensions have been tested.

\section{Structure Description Experimental Results}

Structures under test are shown in Fig.la (microstrip version) and Fig.1b (coplanar line version). They are designed using a probe of a rectangular shape (patch) supported by a dielectric substrate extended into the waveguide through an aperture in the broad wall perpendicularly to the waveguide axis. The analysis by $M A B$ method indicates that there is practically no difference between structures with a dielectric substrate of width equal to the width of a microstrip shielding channel or with a substrate covering the whole waveguide cross-section. So the latter type was used because it can bear a probe in the shape of a patch. The patch on the substrate can face either a backshort (as in Fig. 1) or the waveguide. It has been proved that the first version has a broader band width. The sliding backshort in the test structure is used to optimize the transition bandwidth. For easier access the structure was tested in X-band. A substrate with a thickness of $0.794 \mathrm{~mm}$ and permitivity of 2.35 was used.

The influence of the backshort is shown in Fig.2, where the insertion losses of the waveguide-to-microstrip transition with various backshort positions are plotted. Patch dimensions are $10 \times 6 \mathrm{~mm}$, and its distance from the upper waveguide wall is $1 \mathrm{~mm}$. Strip width is $2.3 \mathrm{~mm}$ this ensures a characteristic impedance of $50 \mathrm{ohms}$ of the microstrip line in the shielding channel with dimensions of $8 \times 5 \mathrm{~mm}$. The broadest bandwidth was obtained with a backshort position (distance from the substrate) of around $5 \mathrm{~mm}$. Dimensions of the shielding channel have a rather weak influence. Another structure with channel dimensions of $4 \times 2.5 \mathrm{~mm}$ and a strip width of $1.93 \mathrm{~mm}$ (for $50 \mathrm{ohms}$ ) was measured, and results were nearly the same. The behaviour of the waveguide-tocoplanar line transition is similar as it can be seen from Fig.2 (the same substrate and patch geometry, strip width $3 \mathrm{~mm}$, slot widths $0.1 \mathrm{~mm}$ - for $50 \mathrm{ohms}$ ).

Several other structures with different patch dimensions have been experimentally tested. All have optimal backshort position in a range from 4 to $6 \mathrm{~mm}$. This fact is documented in Fig. 3 . Measured return losses of various structures with the passband properly adjusted by the backshort are plotted here.

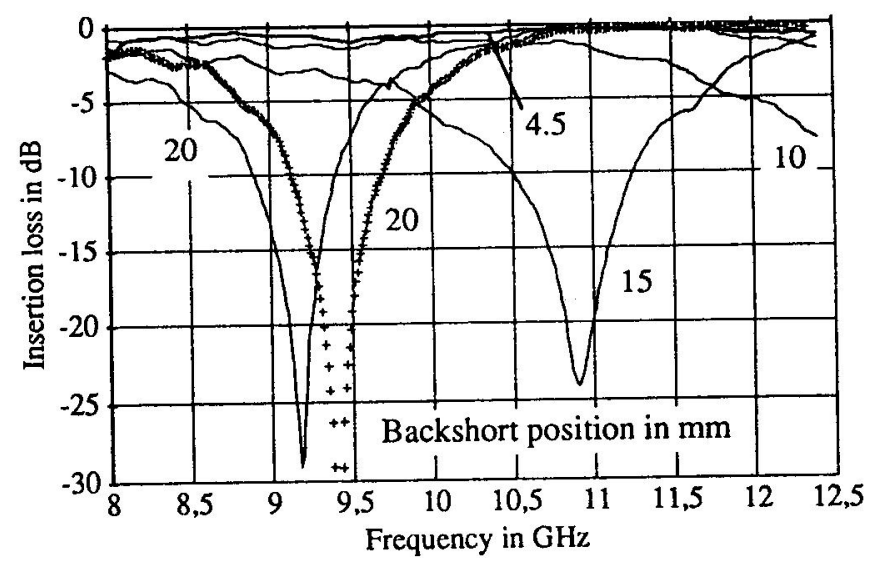

Fig. 2: Insertion losses of the waveguide-to-microstrip transition for various
backshort positions, ++++ waveguide-to-coplonar line transition. 


\section{Transition Analysis and Optimization}

Firstly, the MAB method was used to analyze the transition behaviour. This method is briefly described in [5]. In fact, it is partly similar to a segmentation method. The volume to be analysed is divided into rectangular parallelepipeds (blocks). Scattering matrices of these blocks are determined directly solving the Maxwell's equations in each block independently They are combined to a resulting scattering matrix. This method takes into account the proper structure geometry, but due its complexity, calculation is rather time consuming, and in this case it was not possible to use a net of blocks with sufficient density. However, basic properties of the transition structure have been determined by this method as was mentioned in preceding paragraph.

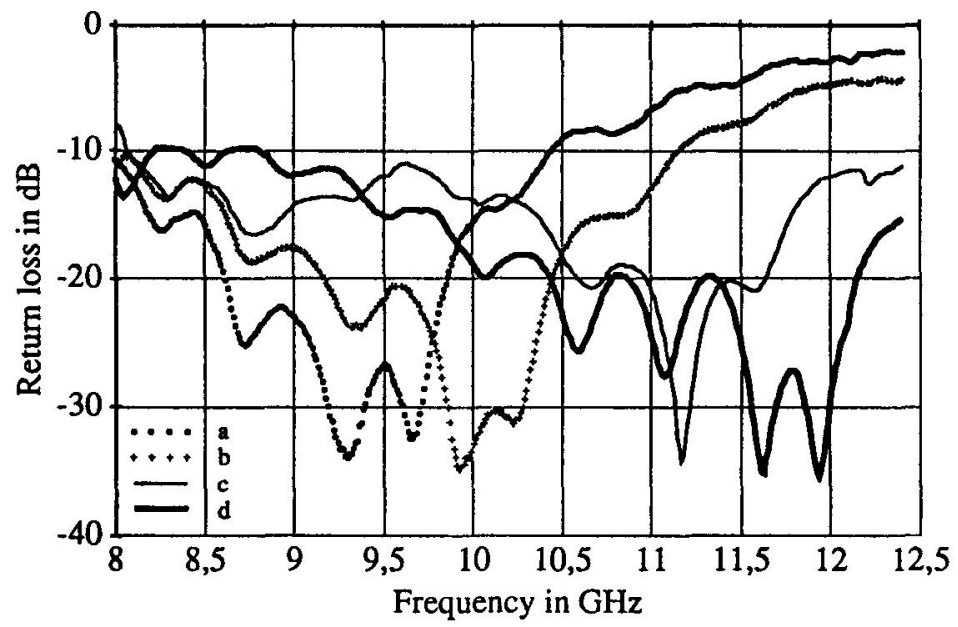

Fig.3: Measured reflection losses of waveguide-to-microstrip transitions with patch dimensions and a backshort distance: a) $14 \times 6 \mathrm{~mm}$, $5 \mathrm{~mm}$, b) $12 \times 6 \mathrm{~mm}, 5 \mathrm{~mm}$, c) $10 \times 6 \mathrm{~mm}, 4.5 \mathrm{~mm}$. d) waveguide-tocoplanar line transition $10 \times 6 \mathrm{~mm}, 5.6 \mathrm{~mm}$.

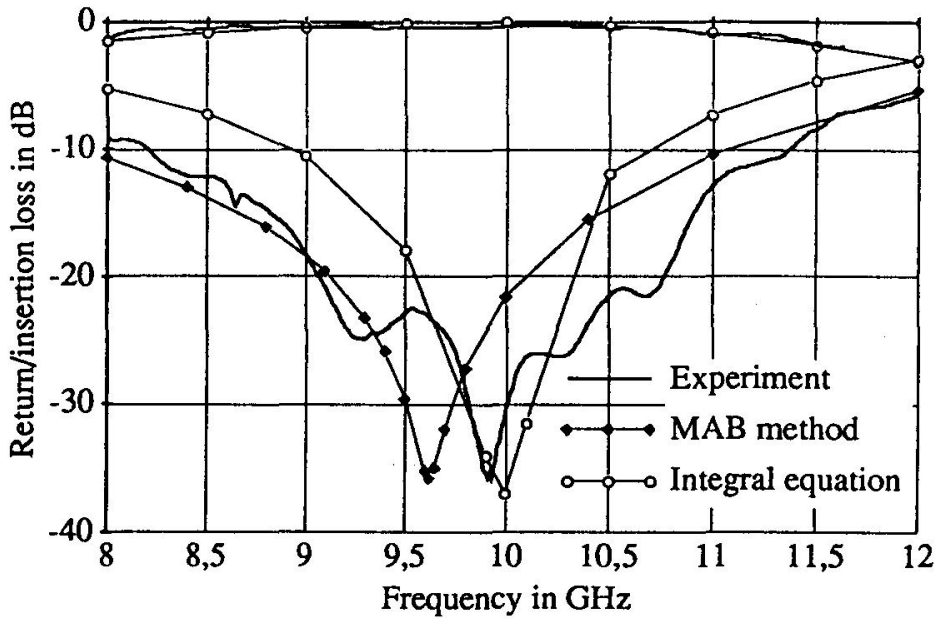

Fig.4: Comparison of $\mathrm{MAB}$ and integral equation methods results with experimental data. Patch dimension are $12 \times 6 \mathrm{~mm}$, backshort distance is $5.2 \mathrm{~mm}$.

Additionally the standard integral equation method was used [4]. The Green's function is determined in the spectral domain. In the process of Fourier retransformation the advantage of a shielded structure (waveguide) was used to substitute integration by summation. Integration is performed in the space domain using the method of moments, and a patch surface current distribution is determined. The field distribution in the aperture of the microstrip shielding channel is not properly described by a sum of waveguide TM and TE modes, so the solution by this method is only approximate. Compared to the MAB method however this solution technique is faster. The comparison of both methods with experiment is shown in Fig.4.

By a simple optimization technique, the patch dimensions can be designed according to a chosen backshort position and a desired transition bandwidth. The error function was determined by the integral equation method. In the case of the waveguide-tomicrostrip transition with the backshort position $5 \mathrm{~mm}$, for return losses lower than $-15 \mathrm{~dB}$ at 8 and $12 \mathrm{GHz}$ and lower than $-20 \mathrm{~dB}$ at $10.2 \mathrm{GHz}$, we obtained patch dimensions of $9 \times 5 \mathrm{~mm}$. Insertion and return losses of this structure are plotted in fig.5. The actual backshort position for the broadest bandwidth is $5.5 \mathrm{~mm}$.

\section{Conclusions}

New versions of the waveguide-to-microstrip line and waveguide-to-coplanar line transitions have been designed and tested. They differ from the standard E-plane transition [2] by the probe in the shape of the rectangular patch. The transition band can be adjusted by the position of the backshort. A waveguide-to. microstrip transition with return losses lower than $-15 \mathrm{~dB}$ from 8 to $11.4 \mathrm{GHz}$ was designed. The behaviour of waveguide-to-coplanar line transitions is similar, but with a slightly narrower bandwidth (Fig.3).

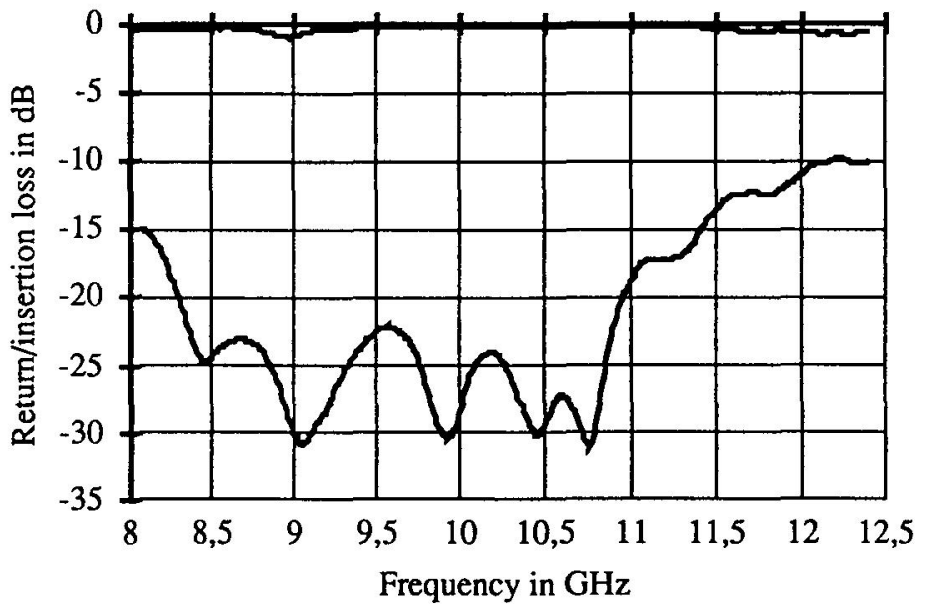

Fig.5: The behaviour of the waveguide-to-microstrip transition with optimal patch dimensions of $9 \times 5 \mathrm{~mm}$. The backshort position is $5.3 \mathrm{~mm}$.

\section{Acknowledgement}

The work was partly done thanks to the support which was given to Dr. J. Machac by the Commision of the European Communities under the scheme of Cooperation in Science and Technology with Central and Eastern European Countries, Research Fellowship Nr. CIPA3510PL920643

\section{References}

[1] Shih, Y.C.: Millimeter-wave device characterisation. Alta Frequnza vol. 58, No. 5-6, 1989, pp. 535-539

[2] Shih, Y.C., Ton, T.N., Bui, L.Q.: Waveguide-to-microstrip transitions for millimeter-wave applications. IEEE Microwave Theory and Techniques Symposium Digest, 1988, pp. 473-475

[3] Ho, T.Q., Shih, Y.C.: Spectral-domain analysis of E-plane waveguide-to-microstrip transitions. IEEE Trans. on Microwave Theory and Techniques, vol. MTT-37, No.2, 1989, pp. 388-392

[4] Mosig, J.R.: Integral equation technique, in Numerical Techniques for Microwave and Millimeter Wave Passive Structures, ed. by Itoh, T.; J. Wiley, New York 1989

[5] Machac, J.: Analysis of discontinuities in waveguiding structures by MAB method. IEE Proc. Part H, Vol. 139, 1992, pp. 351-357. 